\title{
Development of dual frequency gyrotron and high power test of EC components
}

\author{
Y. Oda, K. Kajiwara, K. Takahashi, and K. Sakamoto \\ Japan Atomic Energy Agency, 311-0193 Naka, Ibaraki, Japan
}

\begin{abstract}
In JAEA, development of high-power long-pulse gyrotrons is underway. The output power of the gyrotron was applied for high-power long-pulse tests of the transmission line (TL) and the equatorial launcher (EL) mock up for ITER. The feature of design in the dual frequency gyrotron is the simultaneously satisfying the matching of both frequencies at a window and the same radiation angle at an internal mode convertor for both frequencies. The dual frequency gyrotron was developed and high power operations at $170 \mathrm{GHz}$ and $137 \mathrm{GHz}$ were carried out. The $170 \mathrm{GHz}$ high power experiment of $40 \mathrm{~m}$ length ITER relevant TL was carried out and transmission efficiency and mode purity change caused by long pulse operation were measured. The mock-up model of EL was also tested using $170 \mathrm{GHz}$ gyrotron. The power transmission through the quasi-optical beam line in EL was demonstrated using the full scale mock up model. Furthermore, the high power test results of the transmission components will be summarized.
\end{abstract}

\section{Introduction}

The ITER electron cyclotron heating and current drive (EC H\&CD) system is designed to inject $170 \mathrm{GHz} / 20 \mathrm{MW}$ millimetre wave power into plasma. The system is figured by RF power source, the transmission line (TL), the equatorial port launcher (EL), and the upper port launchers(ULs). RF power sources consist of 24 sets of 1 or $2 \mathrm{MW} / 170 \mathrm{GHz}$ gyrotrons which will be installed in the RF building. A set of two gyrotrons will be operated by a single main high voltage power supply (HVPS) and two sets of acceleration grid power supplies: body power supplies (BPS) and anode power supplies (APS) for triode gyrotron. EU, Russia, and Japan procure 8 gyrotrons[1-3] and EU and India procure 8 and 4 sets of power supply systems. RF power from gyrotron is coupled into TL to transmit from RF building to launchers on the vacuum vessel. TLs are composed of corrugated waveguide system of $63.5 \mathrm{~mm}$ inner diameter including miter bends, polarizers, waveguide switches, and high power dummy loads. The waveguide switch in TL changes the RF power direction to UL or EL. Each line is designed to transmit 1 or $2 \mathrm{MW} \mathrm{RF}$ power for $100 \mathrm{~m}$ distance.[4] $24 \mathrm{TL}$ for each gyrotron system will be procured by US. Single EL procured by Japan and 4 ULs procured by EU will be installed into the equatorial port and the upper port of ITER vacuum vessel.[5,6]

In JAEA, a $170 \mathrm{GHz}$ high power gyrotron with $\mathrm{TE}_{31,8}$ mode demonstrated $1 \mathrm{MW}$ long pulse operation. Now development of high-power long-pulse gyrotrons with higher oscillation mode for two frequency operation is underway.[7] The output power of the gyrotron was applied for highpower long-pulse tests of the transmission line (TL) and the equatorial launcher (EL) mock up for ITER. Transmission line components were examined for high-power long-pulse operation as 
collaboration with General Atomics. The $40 \mathrm{~m}$ length ITER relevant TL which consists of components designed for ITER was coupled with gyrotron output power.[8] For the activity of EL development, the full scale of mock up model was fabricated and the high power test was carried out.[8]

\section{Development of Two Frequency Gyrotron}

After the demonstration of $1 \mathrm{MW}$ long pulse operation using the $\mathrm{TE}_{31,8}$ mode gyrotron, gyrotron development move to higher mode and dual frequency operation. The feature of design in the dual frequency gyrotron is the simultaneously satisfying the matching of both frequencies at a window and the same radiation angle at an internal mode convertor for both frequencies.

The first restriction is at the output window. The disk thickness of the output window should be the integral multiple of the half wavelength in the diamond. The second restriction is in the cavity. The cutoff frequency of the cylindrical cavity must be very close to both main and sub frequencies. The third restriction is compatibility between two frequencies of a mode converter. The mode converter is located just after the cavity that converts the TEm;n oscillation mode to the Gaussian mode. It is a weakly tapered cylindrical dimpled-wall waveguide with a suitable helical cut at the edge. Since the wavelength is small compared with the diameter of the converter, the oscillation mode in the converter can be described in terms of geometric optics (g.o.). The g.o. rays of the TEm;n mode in the cylindrical converter is shown in Fig.1. The same radiation angle for both modes is important, because the g.o. rays are reflected at the same position inside the mode converter and then the optimization of the dimpled wall is straightforward. As a result, similar radiation angle of the Gaussian beam is obtained from the mode converter, which also simplifies the design of the mirrors. Therefore, the gap of the radiation angle defined as should be minimized.

The two completely separate conditions are only satisfied these restriction at the same time. The window thickness of $1.85 \mathrm{~mm}$ for $170 \mathrm{GHz}$ gryotron determined sub frequency as $137 \mathrm{GHz}$ which could transmit the output diamond window. The specific modes $\left(\mathrm{TE}_{31,11}\right.$ and $\left.\mathrm{TE}_{25,9}\right)$ were selected to radiated to the same angle from the cylindrical quasi-optical mode converter to oscillate specific frequencies $(170 \mathrm{GHz}$ and $137 \mathrm{GHz}$ ) with the same cavity design. The internal mode converter was optimized to produce the RF beam from output window with the same radiation angle for both operation frequencies.

The two frequency gyrotron was fabricated and high power short pulse operation was carried out for field profile measurement of the output beam. The RF patterns measured using an IR camera and the calculated RF profiles close to the output window are shown in Fig. 2. As the measured RF patterns show good agreement of the places and the sizes with the calculations, it suggests that the mode converter and the following internal mirrors are working as designed.[7]
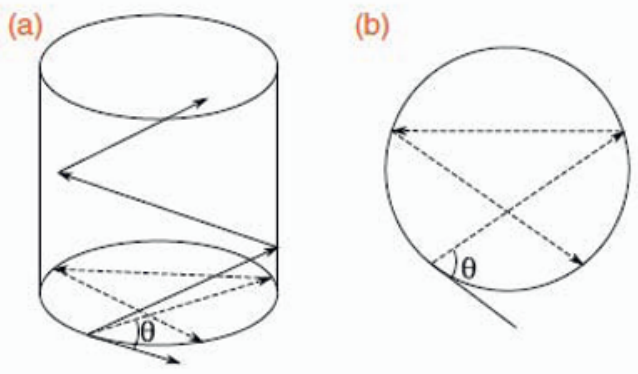

Fig. 1. Geometric optics rays for TEm;n mode in the cylindrical mode converter. (a) Bird's-eye view and (b) top view.[7] 

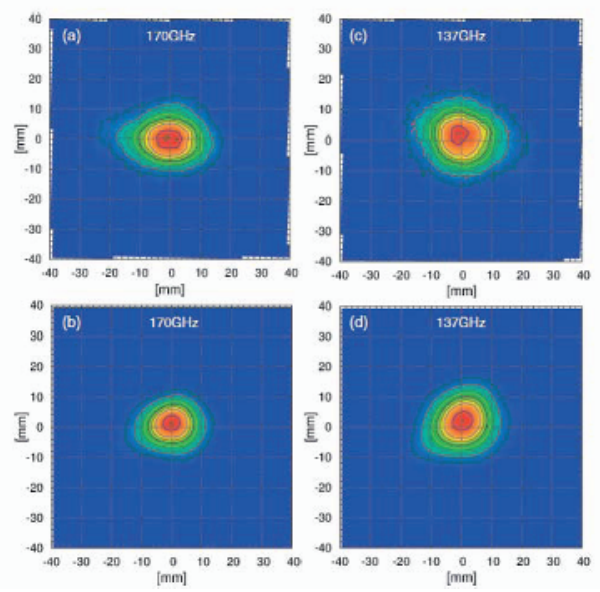

Fig. 2. Linear contour plot of RF power intensities close to the output window for both frequencies. The measured profiles (a) and (c) are compared with the calculations (b) and (d). The measurements are performed using an IR camera.[7]

The gyrotron output beam was injected into the $63.5 \mathrm{~mm}$ circular corrugate waveguide using a matching optical unit (MOU) with phase correcting mirrors designed for the $170 \mathrm{GHz}$ beam. Table 1 lists measured waveguide mode content for both operations. $\mathrm{HE}_{11}$ mode was estimated as $96 \%$ at $170 \mathrm{GHz}$ and $94 \%$ at $137 \mathrm{GHz}$ since MOU mirrors were adjusted at $170 \mathrm{GHz}$. At $137 \mathrm{GHz}$ operation, $1 \%$ of $\mathrm{LP}_{11}$ mode was generated which was caused by beam center offset. Indeed, 137 $\mathrm{GHz}$ beam was well coupled into $\mathrm{HE}_{11}$ mode in waveguide although the mirror design and adjustment was optimized at $170 \mathrm{GHz}$.

RF power from gyrotron was transmitted to a high power long pulse dummy load through TL and long pulse operation was carried out. As a preliminary result, $60 \mathrm{sec}$ duration of pulse was operated at $760 \mathrm{~kW}$ for $170 \mathrm{GHz}$. With same MOU mirror setup, $20 \mathrm{sec}$ duration of pulse was operated at $540 \mathrm{~kW}$ for $137 \mathrm{GHz}$.

Table 1. Measured waveguide mode content at $1 \mathrm{~m}$ waveguide output from MOU.

\begin{tabular}{|l|r|r|}
\hline Mode & \multicolumn{1}{|c|}{$170 \mathrm{GHz}$} & \multicolumn{1}{|c|}{$137 \mathrm{GHz}$} \\
\hline $\mathrm{HE}_{11}$ & $96.0 \%$ & $93.9 \%$ \\
\hline $\mathrm{HE}_{12}$ & $0.6 \%$ & $0.1 \%$ \\
\hline $\mathrm{LP}_{11}$ (even) & $0.3 \%$ & $1.3 \%$ \\
\hline $\mathrm{LP}_{11}$ (odd) & $0.1 \%$ & $0.7 \%$ \\
\hline Others & $3.0 \%$ & $4.0 \%$ \\
\hline
\end{tabular}

\section{High Power Experiment of TL}

\subsection{Mode purity on long distance TL}

Then high power test stand of ITER relevant TL composed of $63.5 \mathrm{~mm}$ corrugated waveguides and TL components designed for ITER was development and examined in JAEA. TL components used in the experiments were fabricated in General Atomic.[8-10] The gyrotron output beam was coupled into TL using MOU and $\mathrm{HE}_{11}$ mode content at inlet of TL was 95\%.[11] The TL test stand includes 4 miter bends, two waveguide switches, and a couple of polarizers. The first waveguide switch (WG-SW-1) selects the short $(7 \mathrm{~m})$ and long $(40 \mathrm{~m}) \mathrm{TL}$. The schematic figure of the TL test stand is shown in Fig.3. 


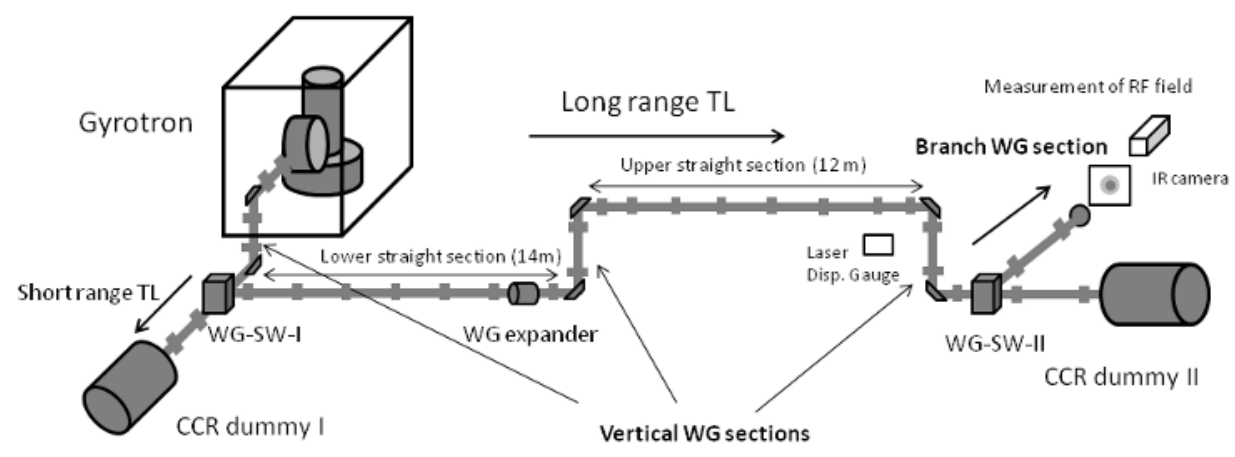

Fig. 3. Schematic figure of JAEA ITER relevant TL high-power test stand.

The mode profile at the outlet of $40 \mathrm{~m}$ TL was measured using a thermal image camera and mode contents were analyzed. After $40 \mathrm{~m}$ transmission, $\mathrm{HE}_{11}$ mode purity was $91 \%$ and the higher order modes was increased. The greatest higher order mode was $\mathrm{LP}_{11}$ (odd) mode which was $4 \%$ although $\mathrm{LP}_{11}$ (odd) mode at $1 \mathrm{~m}$ distance from MOU was $0.8 \%$. The mode content at TL input and output is listed in Table 2. Although the RF beam was successfully coupled into waveguide minimizing high order modes, mode conversion loss occurred by transmission components.

Table 2. Mode contents in TL input and output.

\begin{tabular}{|l|r|r|}
\hline Mode & TL input & TL output \\
\hline $\mathrm{HE}_{11}$ & $95.0 \%$ & $91.1 \%$ \\
\hline $\mathrm{LP}_{11}{ }^{\text {odd }}$ & $0.8 \%$ & $3.5 \%$ \\
\hline $\mathrm{LP}_{11}{ }^{\text {even }}$ & $0.8 \%$ & $0.2 \%$ \\
\hline $\mathrm{HE}_{12}$ & $0.4 \%$ & $1.1 \%$ \\
\hline
\end{tabular}

The measurement result indicates that mode conversion loss by transmission components like miter bends generates $\mathrm{LP}_{11}$ mode and it propagated to TL end. Thereby although good mode purity was achieved at the high efficient beam coupling, the mode conversion loss in TL components becomes source of $\mathrm{LP}_{11}$ mode.

The mode conversion from $\mathrm{HE}_{11}$ mode to $\mathrm{LP}_{11}$ mode by a single miter bend is caused by tilt angle error of the bend mirror. When the mirror included 0.05 deg error, $0.42 \%$ of mode conversion loss reduces $\mathrm{HE}_{11}$ mode content. Thus the mode conversion loss by $40 \mathrm{~m}$ long TL with 7 miter bends is estimated as $3.5 \%$. [10,12] Thereby $\mathrm{HE}_{11}$ mode content at JAEA long range TL output is estimated as $91 \%$ while $95 \%$ of $\mathrm{HE}_{11}$ mode content beam was provided into TL input.

\subsection{Mode conversion by long pulse operation}

Next the mode conversion caused in $40 \mathrm{~m}$ length TL test stand with long pulse operation was measured. TL composed of $63.5 \mathrm{~mm}$ corrugated waveguides. As shown in Fig.2, TL included two waveguide switch and 3 vertical WG sections. Each vertical section was consisted from two miter bends and a $1 \mathrm{~m}$ length waveguide. The miter bend is composed of a flat mirror with the corrugated waveguide section. High power RF dummy loads were connected at the end of TL. The TL had the branch waveguide section and the radiated beam from the branch section was applied for mode purity measurement. A waveguide expander with $27 \mathrm{~mm}$ of axial motion length was settled in the lower straight section of TL. The horizontal displacement of the upper miter bend of the final vertical section was measured by the laser displacement. 
For long-pulse operation test of long range TL, the $170 \mathrm{GHz}$ gyrotron provided $600 \mathrm{~kW}$ RF beam into TL for $169 \mathrm{~s}$. After the end of the operation, WG-SW-II immediately changed the RF direction to branch section for the field profile measurement with short pulse operation and the RF field profiles were measured within $50 \mathrm{~s}$ later. With decline of waveguide temperature, thermal expansion and TL deformation reduced and the TL system recover the initial condition. The displacement increased during the pulse and the maximum displacement was $1.5 \mathrm{~mm}$. This displacement was caused by thermal expansion of the upper long straight section and this deformed the vertical waveguide section.

Figure 4 shows the retrieved field profile at branch section of TL outlet both for before and after the long-pulse operation. Analysis result of mode content showed that $\mathrm{HE}_{11}$ mode content was reduced by $10 \%$. On the other hand, $\mathrm{LP}_{11}$ mode in the transmission power was found to increase by $10 \%$. This indicated that the mode conversion in TL and initial coupling loss in MOU were increased. The mode conversion from $\mathrm{HE}_{11}$ mode to $\mathrm{LP}_{11}$ mode could be caused by waveguide curvature of the deformed waveguide. As same, deformation of MOU structure could generates injection angle of input beam which generates $\mathrm{LP}_{11}$ in TL.

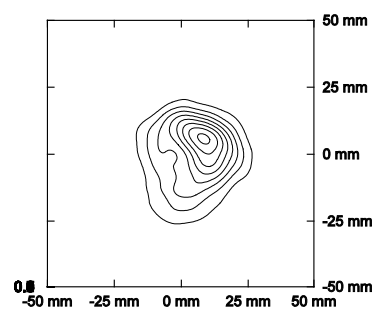

Amplitude

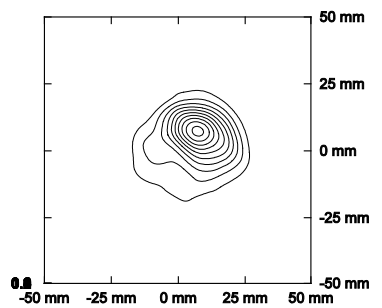

Amplitude

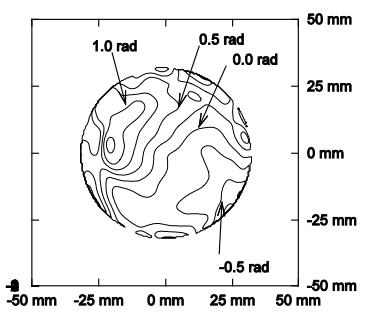

Phase

Before long-pulse operation

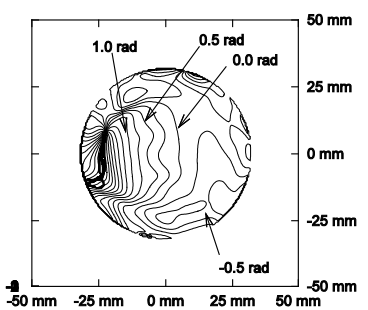

Phase

After long-pulse operation

Fig. 4. Retrieved RF field profile in the waveguide outlet.

Measured mode purity in TL changed significantly, although the JAEA TL test stand achieved 96\% of transmission efficiency.[8] The transmission loss may not increase with thermal deformation of MOU and TL structure because such mode conversion loss generates low mode number HOMs like $\mathrm{LP}_{11}$ mode which has low transmission loss in the waveguide.[13] However the long-pulse operation affects the beam radiation characteristics from the TL outlet significantly and transmission loss in quasi-optical launchers may increase. Thereby it is necessary to reduce the thermal deformation of MOU and TL structure in ITER.

\section{High Power Experiment of EL mock-up}

The mock-up model of EL was connected with TL was tested. The power transmission through the quasi-optical beam line was demonstrated using the high-power gyrotron. The schematic picture of the ITER equatorial EC launcher (EL) is shown in Fig.5. It was designed to inject a $170 \mathrm{GHz}$, 
20MW millimetre wave beam into plasma. EL is consisted of three units and 8 waveguides are connected to each unit. In the launcher unit, 8 millimetre-wave beams were transmitted as a quasioptical (QO) beam through, the focusing mirror, the movable mirror, and opening of the blanket shield modules (BSMs) transmission. The dog-legged waveguide is located in the launcher unit. A toloidal steering range of the beams was settled as $20^{\circ} \leq \Theta_{\mathrm{T}} \leq 40^{\circ}$. The transmission efficiency of the millimetre wave beam on the QO layout is designed as 99.5\% [7]. The issue of QO design is reduction of heat load of steering and focusing mirrors. Thereby the focusing mirror surface was optimized to reduce the heat loads keeping high transmission efficiency of beam bundles in the BSM opening [7,8,14-15]. The centre unit is designed to launch beam bundle for counter current drive.

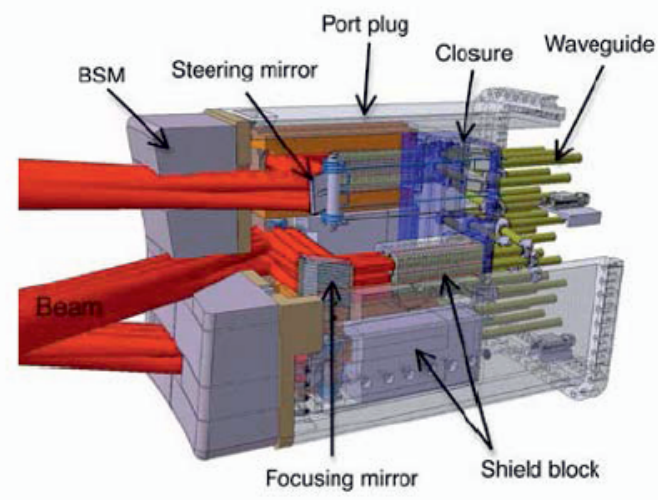

Fig. 5. ITER equatorial EC launcher: The 20MW power is provided by three beam bundles and one of them is injected for the evolution of counter current drive.

Based on this QO design, the full scale EL mock-up was fabricated as shown in Fig.6. The mirrors are located in front of the waveguide structure. The focusing mirror (M1) and the steering mirror (M2) was fabricated as full scale model. In mock-up model, only a single waveguide line was fabricate as an authentic waveguide line with corrugation in the inner surface. For high power experiment of EL mock-up, single beam test with short pulse operation was carried out. Figure 7 shows the power distribution of the millimetre wave beam at the mirrors and the outlet of the EL mock-up measured by using an IR camera. With the steering mirror angle change, the direction of RF beam was successfully changed without beam profile degradation at the outlet of EL mock-up. The measured pattern at the steering mirror agrees with the calculation. However, the measured pattern of the focusing mirror (M1) is inconsistent with the calculation. The high order modes other than $\mathrm{HE}_{11}$ could also be radiated from the waveguide outlet and the measured field patterns at M1 were possibly the mixture of those modes and $\mathrm{HE}_{11}$. This could be a reason of the inconsistency.
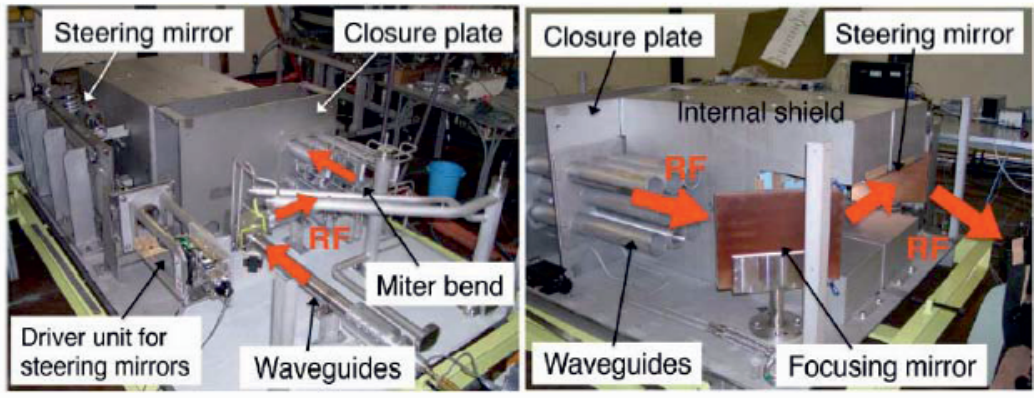

Fig. 6. Photograph of the EL mock-up. [8] 


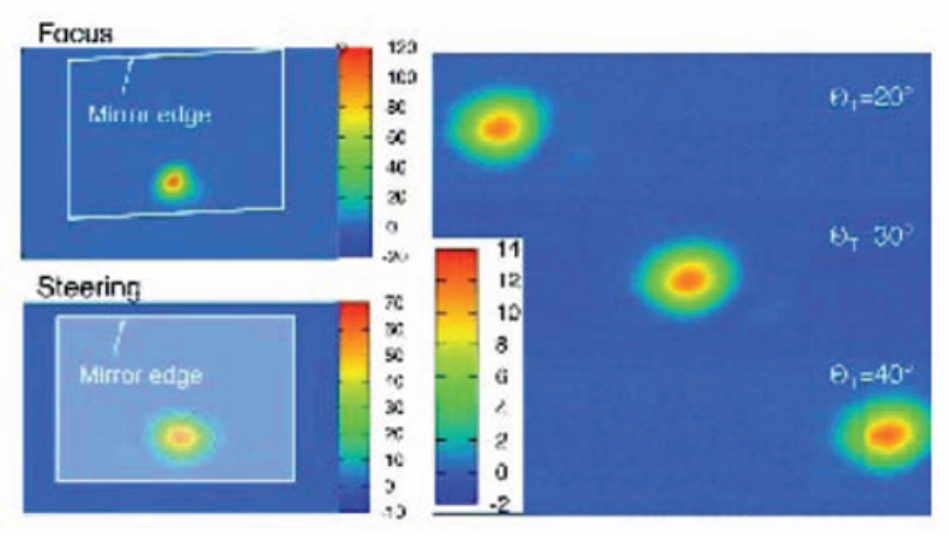

Fig. 7. Measured field pattern of radiated beam at the surface of M1 and M2 and the outlet of the EL mock-up.[8]

\section{Summary}

In JAEA, development of high-power long-pulse gyrotrons with higher oscillation mode for two frequency operation is underway. The two completely separate conditions satisfied the restriction of radiation angle of internal QO launcher and window thickness with specific frequencies $(170 \mathrm{GHz}$ and $137 \mathrm{GHz}$ ) with specific modes $\left(\mathrm{TE}_{31,11}\right.$ and $\left.\mathrm{TE}_{25,9}\right)$. The output $\mathrm{RF}$ beam was designed to radiate from the window with the same radiation angle which allocates to couple the RF beam into TL using a single set of MOU. Then long pulse operation of two frequency gyrotron was successfully carried out with same MOU setup for both frequencies.

ITER relevant TL test stand with $40 \mathrm{~m}$ length was examined for high-power long-pulse operation. After $600 \mathrm{~kW} / 169 \mathrm{~s}$ operation, $\mathrm{HE}_{11}$ mode purity at TL outlet degraded by $10 \%$. This indicated that the mode conversion in TL and initial coupling loss in MOU were increased during the long-pulse operation.

For the activity of EL development, the full scale of mock up model was fabricated and the high power test was carried out. The direction of RF beam was successfully changed with steering mirror operation without beam profile degradation at the outlet of EL mock-up.

\section{References}

1. K. Sakamoto, A. Kasugai, K. Kajiwara, K. Takahashi, Y. Oda, K. Hayashi, N. Kobayashi, "Progress of high power 170 GHz gyrotron in JAEA," Nuclear Fusion, 49, 095019 (2009).

2. M. Thumm, "Recent Development on High-Pwer Gyrotrons," J Infrared Millimeter Terahertz Waves, 32, 241 (2011)

3. G.G Denisov, A.G. Litvak, V.E. Myasnikov, E.M. Tai, V.E. Zapevalov, "Development in Russia of high-power gyrotrons for fusion," Nuclear Fusion, 48, 054007 (2008).

4. F. Gandinit et al., "The EC H\&CD Transmission Line for ITER," Fusion Sci. Tech., 59, 709 (2010).

5. Kajiwara, K. et al. "Design of a high power millimeter wave launcher for EC H\&CD system on ITER, “Fusion Eng. Design, 84, 72 (2009).

6. A. Collazos, V.S. Udintsev, R.Chavan, F. Felici, F. Dolizy, M.A. Henderson, and H. Shidara, "Progress on the ITER upper launcher millimeter wave design and testing," Fusion Sci. Tech., 55, 84 (2009). 
7. K. Kajiwara, Y. Oda, A. Kasugai, K. Takahashi, K. Sakamoto, "Development of DualFrequency Gyrotron with Triode Magnetron Injection Gun,” Appl. Phys. Exp., 4, 126001 (2011).

8. K. Takahashi, K. Kajiwara, Y. Oda, A. Kasugai, N. Kobayashi, K. Sakamoto, J. Doane, R. Olstad, and M. Henderson, "High power millimeter wave experiment of ITER relevant electron cyclotron heating and current drive system," Rev. Sci. Inst., 82, 063506 (2011).

9. R.A. Olstad, J.L. Doane, C.P. Moeller, "ECH MW-level CW Transmission Line Components Suitable for ITER", Fusion Eng. Design, 74, 331 (2005)

10. J. L. Doane, "Design of Circular Corrugated Waveguides to Transmit Millimeter Waves at ITER," Fusion Sci. Tech., 53, 159 (2010)

11. Y. Oda, K. Kajiwara, K. Takahashi K. Sakamoto, "Gyrotron Beam Coupling Method into Corrugated Waveguide," Fusion Sci.Tech. 61, 3, 203 (2012).

12. M. A. Shapiro, E. J. Kowalski, J. R. Sirigiri, D. S. Tax, R. J. Temkin, T. S. Bigelow, J. B. Caughman, and D. A. Rasmussen, "Loss Estimate for ITER ECH Transmissison Line Including Multimode Propagation,” Fsuion Sci. Tech., 57, 196, (2010).

13. B. Plaum, G. Gantenbein, W. Kasparek, M. Thumm, and D. Wagner, "Far Field Caluciations and Measurements of S-bend Deformed Oversized HE11-waveguide Antenna," Int. J. Infrared and Millimeter Waves, 20, 1009 (1999).

14. Takahashi, K. et al. "Improved design of an ITER equatorial EC launcher, “Nucl. Fusion. 48, 5, 054014 (2008).

15. Takahashi, K. et al. "Progress of ITER Equatorial Electron Cyclotron Launcher Design for Physics Optimization and toward Final Design,"Fusion Eng. Design, 86, 982 (2011). 